\title{
$\mathrm{M}|\mathrm{R}| \mathrm{S}$ Internet Journal Nitride Semiconductor Research
}

\section{Improvements in Bis(cyclopentadienyl)magnesium Purity as Determined with Gas Chromatography-Mass Spectroscopy}

\author{
Michael E. Bartram ${ }^{1}$ \\ ${ }^{1}$ Sandia National Laboratories/New Mexico, \\ (Received Tuesday, February 22, 2000; accepted Wednesday, April 26, 2000) \\ Bis(cyclopentadienyl)magnesium ( $\mathrm{MgCp} 2)$ is used commonly as a source for doping nitride \\ materials with magnesium. Increased oxygen incorporation known to accompany the use of $\mathrm{MgCp} 2$ \\ makes the purity of this precursor an important consideration in nitride CVD. Gas chromatography- \\ mass spectroscopy (GCMS) methods have now been developed for the identification of volatile \\ impurities in $\mathrm{MgCp} 2$. Diethylether, an oxygen containing organic compound $\left(\mathrm{CH}_{3} \mathrm{CH}_{2} \mathrm{OCH}_{2} \mathrm{CH}_{3}\right)$, \\ and additional organic impurities were found in the $\mathrm{MgCp} 2$ supplied by three manufacturers. \\ Subsequent refinements in the synthetic processes by these companies have resulted in the \\ availability of $\mathrm{MgCp} 2$ free of ether and other organic impurities as determined by GCMS.
}

\section{Introduction}

The development of the precursors used in CVD (chemical vapor deposition) processes has been facilitated over the years by combining novel synthetic strategies with state-of-the-art analytical techniques such as FTNMR (Fourier transform nuclear magnetic resonance), ICP-MS (inductively coupled plasma mass spectroscopy), ICP-AES (inductively coupled plasma atomic emission spectroscopy), and FTIR (Fourier transform infrared spectroscopy). While these techniques can provide essential information about trace level contaminants, it is not unusual for the characteristic signatures of such impurities to be quite similar to the desired precursor. [1] This difficulty could be circumvented if separation of the individual compounds in a mixture via chromatographic methods could be achieved prior to detection. Unfortunately, the interaction with the stationary phase in the chromatographic column which is responsible for separation [2] can also lead to the decomposition of highly reactive compounds, thus limiting the effectiveness of CVD precursor analysis. For these reasons, the routine use of chromatographic methods has not been part of the rigorous analyses used for quality control in CVD precursor syntheses.

Gas chromatography-mass spectroscopy (GCMS) methods have now been developed for a number of CVD precursors. [1] The key improvements for successful GCMS implementation have been judicious column selection, low column temperature, gas handling and sample introduction appropriate for both the pyrophoric and toxic properties of CVD precursors, the use of chromatographic line-shapes for the recognition of column-induced decomposition, and the use of surrogate compounds as a measure of the analytical species of interest. [1] This paper presents how these have been developed into an effective means for analyzing the volatile impurities in bis(cyclopentadienyl) magnesium (MgCp2) which is used commonly for doping $\mathrm{GaN}$ with magnesium in CVD processes. Bubblers from three manufacturers (companies "A", "B", and "C") were analyzed before and after these companies made improvements in the $\mathrm{MgCp} 2$ synthetic processes.

\section{Experimental}

This study was carried out with a Hewlett Packard 5890 Series II Plus, GCMS using a high resolution capillary column. [2] This was equipped with an electropolished stainless steel gas manifold attached to the column injection head via a sampling valve. The $\mathrm{MgCp} 2 \mathrm{bub}-$ bler was attached as shown in figure 1. GCMS parameters are summarized in Table I.

As with any analytical technique, consideration of the sampling method is a critical factor in ensuring the validity of the results. Certainly, to quantify impurity levels in terms of bulk concentrations, it might be preferable to analyze the $\mathrm{MgCp} 2$ solid directly. However, such an approach is not necessarily the most ideal for first identifying trace level contaminants. It is often prefera- 
ble to pre-concentrate impurities. In the case of condensed phases, promoting the evolution of the impurities out of the bulk and into the gas-phase provides this advantage. [1] For this reason, bubblers were prepared by first evacuating the inert gas over-pressure via the gas manifold on the GCMS. Volatile compounds were then allowed to evolve from the solid $\mathrm{MgCp} 2$ in the closed bubbler for a period of time. For sample extraction, the manifold was evacuated and then the valve connected to the short tube inside the bubbler was opened momentarily. This allowed the manifold and the sampling valve to be filled with volatile compounds present in the bubbler above the $\mathrm{MgCp} 2$ solid. A Baratron gauge mounted on the manifold was used to measure the pressure in the bubbler. The lack of pressure increase monitored as a function of time with the Baratron also confirmed that the manifold did not decompose the sample taken from the bubbler. Therefore, species detected with the GCMS were not artifacts of the method used to sample the bubblers. In fact, this demonstrated that the $\mathrm{Mg}$ precursor is stable in stainless steel tubing for a period of time that is quite long compared to the manifold residence time for GCMS sampling (as well as that of a typical CVD growth run). The sample was then introduced into the column by rotating the sampling valve into the stream of He carrier gas. Mass spectra were acquired continuously as compounds eluted from the column into the MS inlet. Although compounds were identified based on a complete mass spectrum, the chromatograms are presented in terms of the total ion current for simplicity. Specifically, the total ion current refers to the sum of the signals acquired at each mass/charge ratio within a complete mass spectrometer scan taken at a given retention time.

\section{Results}

The chromatogram of the first $\mathrm{MgCp} 2$ bubbler from Company A (designated as bubbler A1) is shown in figure 2 . The number of peaks in the chromatogram suggest that the bubbler contained a mixture of 6 compounds in addition to $\mathrm{N}_{2}$. The chromatographic resolution [2] (baseline peak separation) was sufficient to observe a unique mass spectrum with constant ion ratios through out a given peak. In addition to $\mathrm{MgCp} 2$ (observed as a cyclopentadiene surrogate), propane, diethylether, methylcyclopentadiene (observed as a surrogate for $\mathrm{Mg}(\mathrm{MeCp}) 2$ possibly), 3a,4,7,7a-tetrahydro(M)4,7-methano-1H-indene (referred to commonly as a cyclopentadiene "dimer"), and dodecane were assigned to each peak. [3] The bubbler was immersed in hot water $\left(\sim 50{ }^{\circ} \mathrm{C}\right.$ for $15 \mathrm{~min}$.) to promote the evolution of volatile components from the solid. This lowered the concentration of all but the cyclopentadiene to below the GCMS detection limit as shown in figure 3. In addition, the cyclopentadiene peak was decreased substantially and the pressure observed in the sampling manifold was lowered to that of the vapor pressure of MgCp2 ( 40 mTorr [4]). Importantly, evacuation did not result in a significant loss of $\mathrm{MgCp} 2$ from the bubbler.

Collectively, these observations suggest that the greater absolute intensity of the cyclopentadiene peak in figure 2 (A1) has contributions from $\mathrm{MgCp} 2$ decomposition in both the bubbler and in the GC injector and column. Decomposition in the mass spectrometer of the GCMS is unlikely since the published mass spectrum of MgCp2 using a break-tube for sample introduction [5] is quite different from cyclopentadiene alone. Further, the slight plateau in intensity near the baseline is the characteristic line-shape for the on-going decomposition of a reactive compound in the chromatographic column. [1] Although this can be a serious complication, [1] the plateau peak shape is not a encumbrance in this study since it does not obscure the detection of the impurities accompanying $\mathrm{MgCp} 2$. The fact that the cyclopentadiene peak is quite large relative to the plateau implies that the large majority of $\mathrm{MgCp} 2$ decomposes in either the injector or at the beginning of the column immediately. Therefore, the presence of additional cyclopentadiene (relative to figure 3 as seen by the signal-to-noise) is due to $\mathrm{MgCp} 2$ decomposition in the bubbler. An alternative column might cause less $\mathrm{MgCp} 2$ decomposition given a weaker interaction, but at the cost of chromatographic resolution (separation of the individual components would be less efficient). Finally, the presence of the cyclopentadiene dimer results from the well-known "4+2 Diels-Alder addition reaction" in which two cyclopentadiene rings react together at room temperature. [6]

If not involved in the synthesis directly, then diethyl ether is likely to be involved in the post-synthesis extraction and/or recrystallization of $\mathrm{MgCp} 2$. Volatile solvents such as this are used quite commonly in these final isolation and purification steps. Although, a very short evacuation time (or flowing carrier gas) was sufficient to lower the contaminants in this bubbler to below the GCMS detection limit, concerns about residual ether contributing to oxygen impurity incorporation in $\mathrm{Mg}$ doping applications prompted the development of a new ether-free process for $\mathrm{MgCp} 2$ synthesis.

The chromatogram of Company A's new MgCp2 process is shown in figure 2 as bubbler A2. The hydrocarbon impurities are depicted by each peak. It should be noted that the bubbler was submitted for analyses without the preconditioning that Company A would do normally to remove residual solvent left over from the final synthesis step. This was done for the specific purpose of observing the worst case possible. In fact, the chromatogram shown in figure 3 is representative of the effect that evacuation after immersion in hot water has on the bubbler contents; only $\mathrm{MgCp} 2$ is left remaining 
in the bubbler. This procedure is the near equivalent to flowing carrier gas in a preconditioning step when the bubbler is first mounted on a CVD system. Certainly, the residual hydrocarbons were removed quite well, showing that this level of in-situ purification can be accomplished easily and quickly with standard CVD gas handling capabilities. Importantly, the residual hydrocarbons did not continue to evolve, as judged with GCMS over the course of approximately two weeks, showing that this one-time procedure removed the hydrocarbons from the solid completely. Decomposition of $\mathrm{MgCp} 2$ into cyclopentadiene derivatives and dimers was detected, however these could be removed in a few minutes by evacuation or by flowing carrier gas at room temperature.

GCMS analysis of $\mathrm{MgCp} 2$ from Company $\mathrm{B}$ also resulted in the detection of ether, as shown in figure 4 in bubbler B1. In this case, the dimethyl derivative of cyclopentadiene was observed, suggesting the presence of $\mathrm{Mg}(\mathrm{Me} 2 \mathrm{Cp}) 2$ along with $\mathrm{MgCp} 2$. Two ether-free processes were then tested. The total ion chromatograms given in figure 4 reveal that both bubblers (B2 and B3) have mixtures of long-chain hydrocarbons in a variety of structural forms (isomers) in addition to $\mathrm{MgCp} 2$. In addition, there is no evidence of any oxygen containing compounds in either bubbler. Both B2 and B3 were examined for ease of in-situ purification by warming ( $50{ }^{\circ} \mathrm{C}$ ) the bubblers and then evacuating. Regardless of the duration of this procedure, hydrocarbon impurities continued to evolve out of the solid $\mathrm{MgCp} 2$ in bubbler B2. Conversely, all hydrocarbon impurities were removed from bubbler B3 in less than $30 \mathrm{~min}$ and did not reappear over the 2 week time period monitored with GCMS. A chromatogram of this result is equivalent to that shown in figure 3. Clearly, the process used for synthesizing the $\mathrm{MgCp} 2$ in bubbler B2 also traps solvents etc. which then continue to escape from the solid for a significant amount of time. Therefore, although hydrocarbons are present at detectable level in bubbler B3 initially, the synthetic process does not lead to the occlusion of solvents during the final recrystallization of $\mathrm{MgCp} 2$. This allows in-situ purification to be carried out easily and efficiently with a standard CVD gas handling system.

$\mathrm{MgCp} 2$ obtained from Company $\mathrm{C}$ did not provide any evidence for ether. Interestingly, bubbler $\mathrm{C} 1$ in figure 5 had a mixture of hydrocarbons quite like that observed in bubbler B2 (figure 4), although it was of much greater magnitude. Similarly, extended evacuation aided by warming the bubbler was not effective at removing the hydrocarbon impurities completely. How- ever, the $\mathrm{MgCp} 2$ received in a second bubbler (C2) from Company $\mathrm{C}$ was quite pure as shown in figure 5 . Warming the bubbler did not result in the detection of significant levels of hydrocarbon impurities.

\section{Summary}

GCMS operating conditions can be chosen that yield useful analyses of reactive CVD precursors such as $\mathrm{MgCp} 2$. The use of low temperatures and surrogate detection combined with line-shape considerations has allowed GCMS to aid in the selection of new processes for ether-free syntheses of $\mathrm{MgCp} 2$. Also, procedures for in-situ purification and the removal of decomposition products have been verified with this study. Under no condition, did significant loss of $\mathrm{MgCp} 2$ occur as a result of the evacuation-warming procedures described in this report.

It should be emphasized that the $\mathrm{MgCp} 2$ chromatogram yielded upon purification for each company was equivalent to that shown in figure 3 . Therefore, each of the three companies are now capable of producing $\mathrm{MgCp} 2$ that is exceptionally pure. To obtain $\mathrm{MgCp} 2$ of this quality, simply specify that in addition to passing all of the other standard analytical tests, the $\mathrm{MgCp} 2$ has to be free of ether processing and other hydrocarbon impurities, as verified with GCMS.

\section{ACKNOWLEDGMENTS}

The enthusiastic support and contribution of $\mathrm{MgCp} 2$ for this study from Larry Guilbault and Bob Stennick of Rohm Haas, Ravi Kanjolia and Phil McGraw of Epichem Inc., and Ephraim Honig and Frank Wagner of Strem Chemical, Inc. is appreciated greatly. Sandia is a multi-program laboratory operated by Sandia Corporation, a Lockheed Martin Company, for the United States Department of Energy under Contract DE-AC04-94AL85000.

\section{REFERENCES}

[1] M.E. Bartram, unpublished.

[2] R.L. Grob, Ed. "Modern Practice of Gas Chromatography", 2nd Edition, John Wiley \& Sons, New York, 1985

[3] NIST/EPS/NIH Mass Spectral Library, NIST Mass Spectral Search Program V1.5 7/19/96, U.S. Department of Commerce

[4] Materials Safety Data Sheets from Morton International, Inc. and Strem Chemical, Inc

[5] L. Friedman, A. P. Irsa, G. Wilkinson, J. Am. Chem. Soc. 77, 3689 (1955).

[6] J. March , "Advanced Organic Chemistry: Reactions, Mechanisms, and Structure", 2nd Edition, McGraw Hill, Inc. New York, 1977, p761-2 


\section{FIGURES}

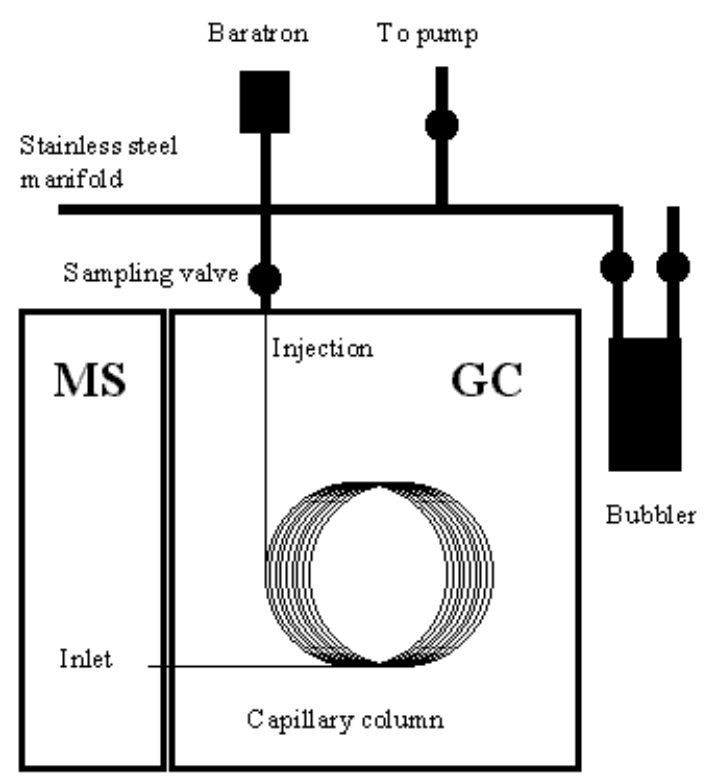

Figure 1. Stainless steel gas handling manifold attached to the injection head of a Hewlett Packard 5890 Series II Plus GCMS. All zones (sampling valve, injection head, capillary column, and source inlet) were maintained at $75^{\circ} \mathrm{C}$.
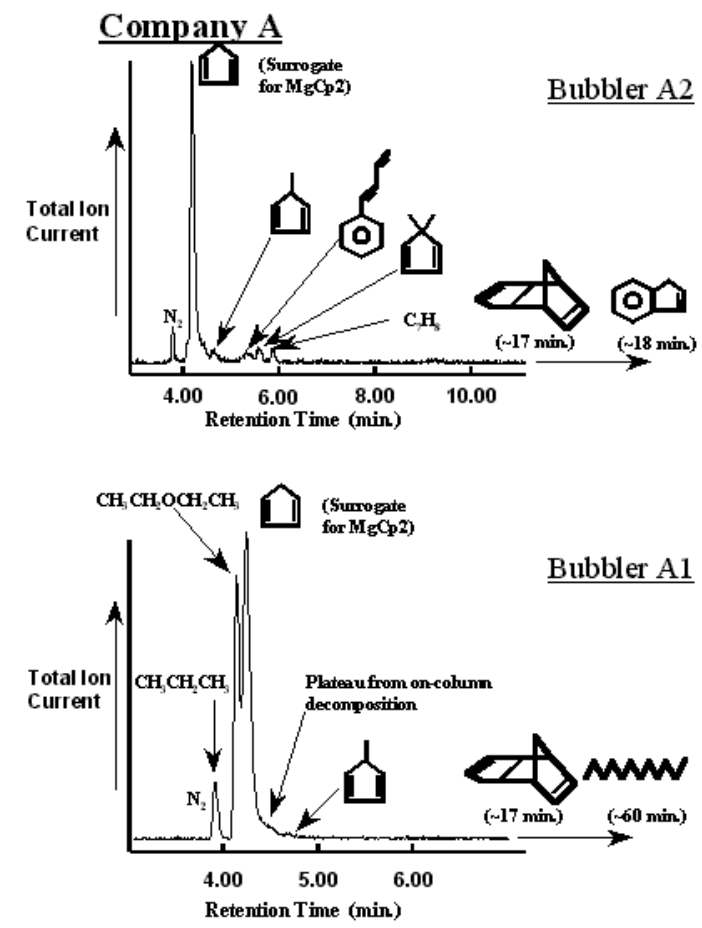

Figure 2. Chromatograms of $\mathrm{MgCp} 2$ in bubblers from Company "A". Different processes were used to synthesize the $\mathrm{MgCp} 2$ in each bubbler. The $\mathrm{MgCp} 2$ in bubbler A2 was produced without using diethyl ether $\left(\mathrm{CH}_{3} \mathrm{CH}_{2} \mathrm{OCH}_{2} \mathrm{CH}_{3}\right)$. Impurities in each bubbler could be removed by evacuating for a short period of time ( $\sim 15 \mathrm{~min}$.) at elevated temperatures $(\sim 50$ $\left.{ }^{\circ} \mathrm{C}\right)$ as shown in figure 3 .

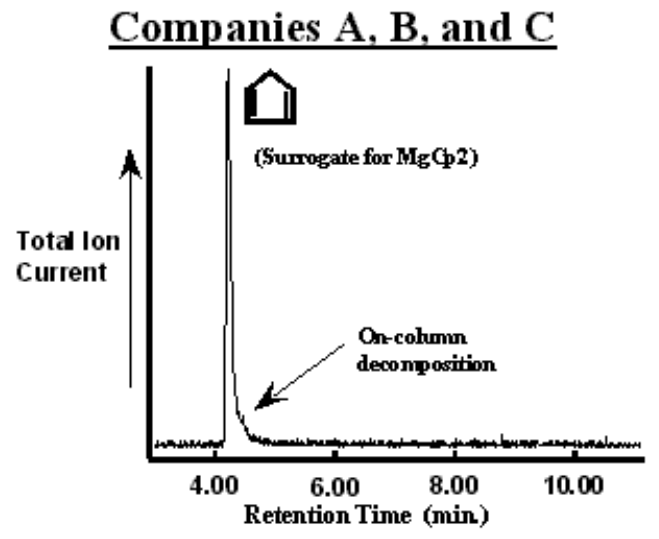

Figure 3. A chromatogram of pure $\mathrm{MgCp} 2$ obtained after evacuating bubbler A1 in figure 2. Identical chromatograms were obtained after evacuating bubbler $\mathrm{A} 2$ as well as from the optimized $\mathrm{MgCp} 2$ processes of companies B and C (bubblers $\mathrm{B} 3$ and $\mathrm{C} 2$ described in figures 4 and 5). 

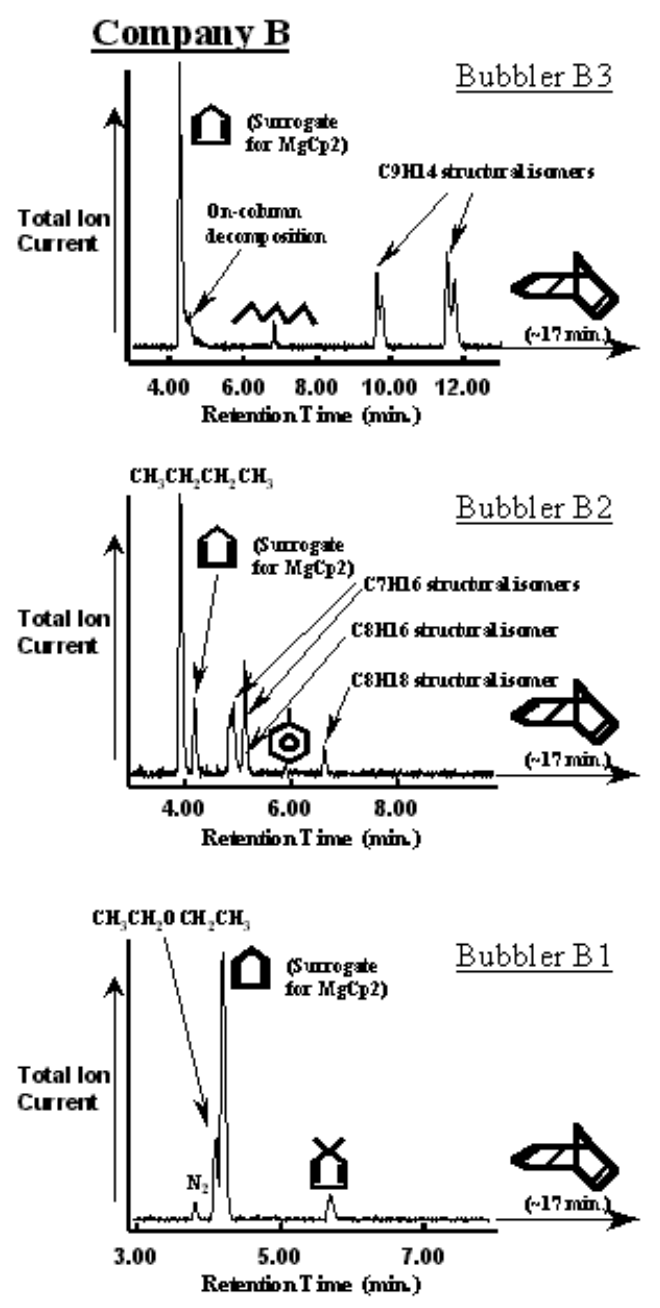

Figure 4. Chromatograms of $\mathrm{MgCp} 2$ in bubblers from Company "B". Different processes were used to synthesize the $\mathrm{MgCp} 2$ in each of the three bubblers. Neither of the processes for bubblers B2 and B3 used diethyl ether. However, only B3 could be purified fully by evacuation. The final result is identical to the chromatogram shown in figure 3 .

\section{Company C}
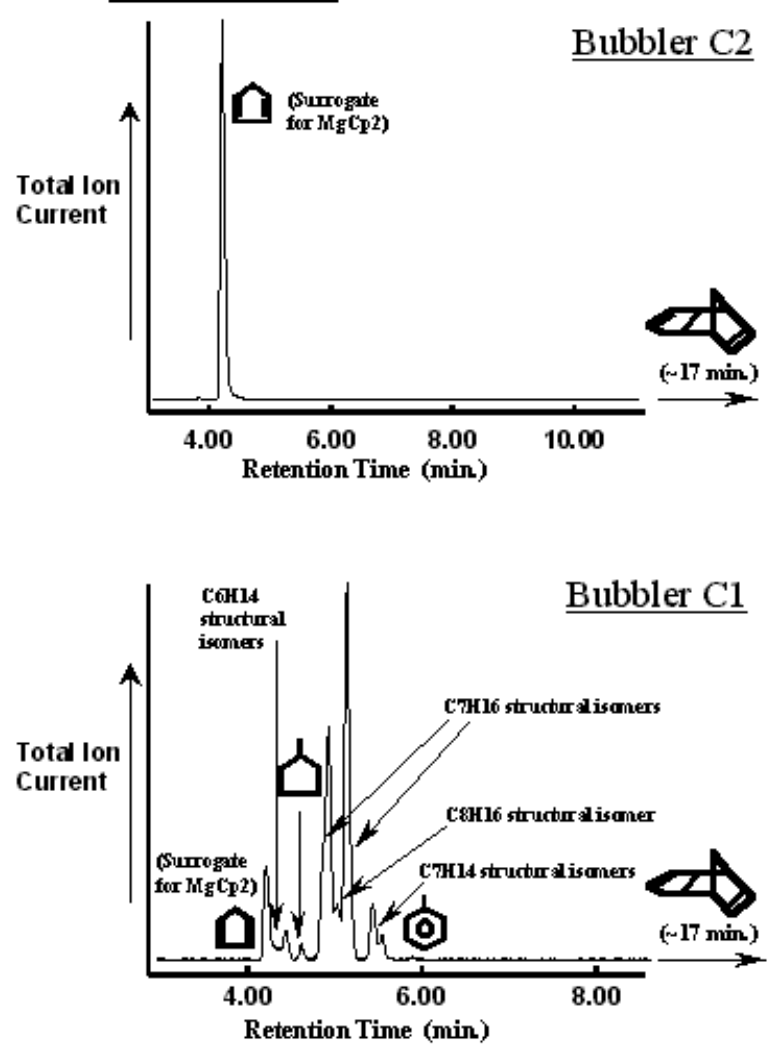

Figure 5. Chromatograms of $\mathrm{MgCp} 2$ in bubblers from Company " $\mathrm{C}$ ". Different processes were used to produce the $\mathrm{MgCp} 2$ in bubblers $\mathrm{C} 1$ and $\mathrm{C} 2$. No hydrocarbon impurities were detected in $\mathrm{C} 2$.

\section{TABLES}

Table I. GC Parameters for MgCp2 analyses

\begin{tabular}{|l|l|}
\hline Injection time-span: & $\begin{array}{l}18 \mathrm{~s} \text { (before returning the sampling } \\
\text { valve to the normal position) }\end{array}$ \\
\hline Column type: & $\begin{array}{l}\text { Non-polar (100\%) high-resolution } \\
\text { capillary (HP-1) (60m long, .250 } \\
\text { mm id, .25um film thickness) }\end{array}$ \\
\hline Flow rate: & $\begin{array}{l}20 \mathrm{~cm} / \mathrm{s} \mathrm{He} \text { electronic grade } \\
(99.999 \%) \text { at } 20 \text { psi }\end{array}$ \\
\hline Temperature: & $\begin{array}{l}75^{\circ} \mathrm{C} \text { in all zones (sampling valve, } \\
\text { injection, column, and MS inlet) }\end{array}$ \\
\hline Inlet purge: & Yes \\
\hline Elution time (complete): & $\begin{array}{l}<20 \text { min. except for dodecane }(~ 60 \\
\text { min.) }\end{array}$ \\
\hline MS scan range: & mass / charge 1 - 300 \\
\hline MS scan rate: & Full range scanned every 1/2 s \\
\hline
\end{tabular}

